\title{
Tapentadol prolonged release for severe chronic cancer-related pain: effectiveness, tolerability, and influence on quality of life of the patients
}

This article was published in the following Dove Press journal: Journal of Pain Research

22 December 2014

Number of times this article has been viewed

\section{Artur Schikowski' \\ Doris Krings ${ }^{2}$ \\ Karla Schwenke ${ }^{2}$}

'Neurology and Specialist Pain Therapy, Specialist Center Düsseldorf, Düsseldorf, ${ }^{2} \mathrm{Grünenthal} \mathrm{GmbH}$, Aachen, Germany
Correspondence: Artur Schikowski Facharzt Zentrum Düsseldorf, Neurologie und Spezielle Schmerztherapie, Friedrichstrasse 13, 40217 Düsseldorf, Germany Tel +49 2II 3844780 Fax +49 2II 38447814 Email artur.schikowski@t-online.de
Background: Clinical trials have shown the efficacy and good tolerability of tapentadol prolonged release (PR) for severe chronic pain of different etiologies. This study investigated the influence of tapentadol PR on pain control and quality of life of patients with severe chronic cancer-related pain in routine clinical practice in Germany.

Patients and methods: During a 3-month observation period, 45 physicians (mainly palliative care specialists) documented dosage and tolerability of tapentadol PR, previous and concomitant analgesic treatment, pain intensity, pain-related restrictions of daily activities and quality of life, and general state of health of 123 patients with chronic cancer-related pain in the context of a prospective noninterventional study.

Results: All patients (mean age 63.9 13.2 years, 93.5\% in constant pain) had received analgesic long-term treatment ( $42.3 \%$ strong opioids) prior to the start of tapentadol PR treatment. During the observation period, tapentadol PR significantly reduced the average pain intensity by 2.4 points (from a mean $6.1 \pm 1.7$ to $3.7 \pm 2.0, P<0.001$ ); half of the patients $(52 \%)$ achieved a pain score $\leq 3$ at the end of observation. At the same time, mental and emotional well-being, pain-related impairments of daily activities, sleep quality, and quality of life improved, while the overall intake of analgesic concomitant medication could be reduced. Improvements in general state of health were significant $(P<0.001)$. Overall, tapentadol PR was well tolerated.

Conclusion: Good pain control with tapentadol PR was accompanied by markedly reduced pain-related mental and physical burden and quality of life improved. Overall, the general state of health of these patients with chronic cancer-related pain improved significantly despite the underlying illness.

Keywords: chronic cancer-related pain, tapentadol PR, pain treatment, quality of life, daily clinical practice

\section{Introduction}

In cancer patients, pain is a significant problem markedly contributing to impairments of daily activities and quality of life. ${ }^{1,2}$ Even mild pain can greatly interfere with patients' daily life. ${ }^{3}$ Opioids play an important role in the treatment of chronic cancer-related pain; ${ }^{4,5}$ their side-effect profile (gastrointestinal and central nervous system disorders) can however result in insufficient compliance or withdrawal from treatment. ${ }^{6}$ The prolonged-release (PR) dosage form of the centrally acting analgesic tapentadol (Palexia ${ }^{\circledR}$ retard; Grünenthal GmbH, Aachen, Germany) is indicated for the treatment of adult patients with severe chronic pain that can only be managed adequately with opioid analgesics. In contrast to classical opioids, this medication combines two mechanisms of action in one molecule: $\mu$-opioid receptor (MOR) binding and noradrenaline reuptake 
inhibition (NRI). Both mechanisms have been shown to contribute synergistically to the analgesic efficacy of tapentadol PR, while at the same time reducing typical opioid side effects, such as nausea, vomiting, and gastrointestinal inhibition. ${ }^{7}$ Efficacy and good tolerability of tapentadol PR in the treatment of severe chronic nonmalignant pain have already been demonstrated in clinical studies ${ }^{8,9}$ and under routine clinical practice conditions. ${ }^{10,11}$ Results in the treatment of cancer-related pain have also been published: ${ }^{12-17}$ in clinical studies, analgesic efficacy of tapentadol PR was shown to be equivalent to oxycodone controlled release ${ }^{13}$ or morphine controlled release, ${ }^{14}$ and demonstrated a more favorable gastrointestinal tolerability profile. ${ }^{13,14}$ Marked relief of cancer-related pain was also reported during 3-month treatment in routine clinical practice. ${ }^{17}$

Apart from pain control, the restoration or preservation of quality of life, in particular at advanced cancer stages, needs to be taken into account. This study thus documented not only changes in pain parameters during routine administration of tapentadol PR in patients with severe chronic cancer-related pain but also included standardized, self-reporting questionnaires regarding pain-related impairments in daily life and quality of life.

\section{Patients and methods Study design and patients}

This prospective, noninterventional study was carried out by 45 physicians in private practice, mainly palliative care specialists (19 anesthetists, 14 specialists for internal medicine, four oncologists, four general practitioners, four other specialists) from March 2012 to February 2013 in accordance with section 4, subsection 23, clause 3 and section 67 , subsection 6 of the Medicinal Products Act of the Federal Republic of Germany. The study objective was to investigate routine administration of tapentadol PR for severe chronic cancer-related pain that can only be adequately treated with opioid-containing analgesics. Only patients for whom treatment with tapentadol PR was planned (irrespective of this study) could participate. All treatment decisions were solely at the discretion of the treating physician. Tapentadol PR was prescribed in accordance with the summary of product characteristics, depending on previous pain treatment and pain severity. ${ }^{18} \mathrm{~A}$ dose adjustment within 3 days was recommended in cases of insufficient pain relief.

\section{Data collection}

Data were documented by the physicians during consultation at baseline examination, during treatment follow-up after
4-6 weeks, and at the end of observation after approximately 3 months. Documentation included demographic data, cancer diagnosis, tumor stage and grading, pain diagnosis and pain type, concomitant diseases and previous analgesic treatment, reasons for prescribing tapentadol PR, tapentadol dosages, and analgesic and other concomitant medications during treatment.

Pain intensity (lowest, average, and highest) during the last 3 days was rated on an 11-point numerical rating scale (NRS11 ; from $0=$ no pain to $10=$ worst imaginable pain). ${ }^{19}$ Functional status of the patients was determined by the physicians using the Karnofsky physical performance score ${ }^{20}$ (scale in 10-point steps from $100=$ normal, no complaints, no evidence of disease, to $0=$ death) and the Eastern Cooperative Oncology Group functional status ${ }^{21}$ (from $0=$ asymptomatic to $5=$ death).

In order to rate the state of their health, patients completed the EuroQoL group's EQ-5D questionnaire (version 3L), which inquires about the five dimensions of mobility, selfcare, usual activities, pain/discomfort, and anxiety/depression, for each dimension choosing from the answer options "no problems", "some problems", and "extreme problems". ${ }^{22}$ In addition, state of health was marked by the patients with a cross on a visual analog scale from $0=$ best imaginable state to $100=$ worst imaginable state.

Furthermore, patients used two standardized self-reporting instruments: the German Pain Questionnaire (for baseline evaluation) and the German Pain Diary (at treatment follow-up and at the end of observation). ${ }^{23}$ In addition to the NRS-11, these questionnaires include the following psychometric scales: the modified Pain Disability Index ${ }^{24}$ (mPDI) for quantification of pain-related impairments in daily activities (single score $0=$ no impairment to $10=$ complete impairment, sum score $>43=$ severely affected), the Marburger Fragebogen zum habituellen Wohlbefinden (MFHW [Marburg questionnaire on habitual health findings] $)^{25}$ to determine habitual well-being (from $0=$ worst possible state to $5=$ best possible state), the Hospital Anxiety and Depression Scale ${ }^{26}$ (HADS) to evaluate anxiety and depression ( sum score $1-7=$ normal, $8-10=$ mildly affected, 11-21=moderately to severely affected), the short form $(\mathrm{SF}-12)^{27}$ of the SF-36 Health Survey to assess health-related quality of life, and a questionnaire to document pain-related impairments in quality of life (Quality of Life Impairment by Pain [QLIP] inventory, ${ }^{23}$ sum score $0=$ total impairment to $40=$ no impairment, $\leq 20=$ severe impairment).

During baseline examination, physicians and patients agreed on a pain-intensity target (NRS-11) for the end of observation (individual treatment target). Physicians documented all adverse drug reactions (ADRs) that occurred during the observation period. 


\section{Response criteria}

Treatment success regarding pain reduction was evaluated using seven criteria for the parameter "average pain intensity": pain-intensity score $\leq 3$ (NRS-11), pain-free state (score of 0, NRS-11), attainment of the individual treatment target, pain reduction of at least 2 points (NRS-11) compared to baseline, pain reduction $>30 \%$ compared to baseline, pain reduction $>50 \%$ compared to baseline, and pain reduction $>70 \%$ compared to baseline.

\section{Statistical analysis}

Collected data were processed with double data entry by O.Meany MD\&PM GmbH (Nuremberg, Germany) using the data-management program FormPro ${ }^{\circledR}$ version 5.1, and checked for completeness, consistency, and plausibility. The case report forms of all 123 prospectively documented patients were included in the effectiveness analysis (carried out by O.Meany MD\&PM GmbH using the statistical program PASW Statistics for Windows version 18.0) and in the safety and tolerability analysis (Pharm-Soft Dr B Rodust $\mathrm{GmbH}$, Ascheberg, Germany). Descriptive $P$-values for the changes over the course of the study in pain intensities and health state were determined with the paired $t$-test. A total of $65 \%$ of the patients answered at least one pain questionnaire/pain diary; however, only $26 \%$ of all patients completed all three documents. These data were analyzed with the "last observation carried forward" method (LOCF). ADRs were encoded with the Medical Dictionary for Regulatory Activities $^{\circledR}$ (MedDRA, version 16.0).

\section{Results}

Table 1 lists the demographic and clinical characteristics of the 123 patients. Nearly $60 \%$ were older than 60 years (59.4\%); the most common primary cancer locations were breast (26.8\%) and male genital organs (19.5\%). Most tumors were solid (83.7\%), $32.5 \%$ of those were at stage $\mathrm{T} 3 / \mathrm{T} 4,50.4 \%$ with spread to regional lymph nodes (N1/N2), and 55.3\% with distant metastases (M1). Sixty-five percent of the patients had had surgery because of the tumor, $68.3 \%$ had received chemotherapy, and $43.9 \%$ radiotherapy. Comorbidities were reported for $69.1 \%$; hypertension was listed most frequently (44.7\%). Patients' ability to function was impaired to varying degrees, with a mean Karnofsky status at baseline of $62.7 \pm 17.2$; a third $(33.3 \%)$ required considerable assistance and frequent medical care (score of $\leq 50$ ). A total of $13.8 \%$ were only capable of limited self-care (Eastern Cooperative Oncology Group status 3). Patients received mainly outpatient
Table I Baseline demographic and clinical data of the patient population $(n=123)$

Sexa

Male $63(51.2 \%)$

Female $59(48.0 \%)$

Age (years) ${ }^{\mathrm{b}} \quad 63.9 \pm 13.2$

Body mass index $\left(\mathrm{kg} / \mathrm{m}^{2}\right)^{\mathrm{b}} \quad 24.3 \pm 4.6$

Type of cancer (ICD-10)

C00-C97 Malignant neoplasms $\quad$ II4 (92.7\%)

C50 Breast $33(26.8 \%)$

C60-C63 Male genital organs 24 (19.5\%)

C30-C39 Respiratory and intrathoracic organs $\quad 18$ (14.6\%)

C15-C26 Digestive organs $\quad 10(8.1 \%)$

C8I-C96 Lymphoid, hematopoietic, and related $\quad 10(8.1 \%)$

tissue, stated or presumed to be primary

C64-C68 Urinary tract $6(4.9 \%)$

Not specified $13(10.6 \%)$

D37-D48 Neoplasms of uncertain or unknown behavior $\quad 2(1.6 \%)$

No data

$7(5.7 \%)$

Duration of pain ${ }^{\mathrm{a}}$

$<$ l year $29(23.6 \%)$

$\geq \mathrm{I}<5$ years $\quad 65(52.9 \%)$

$\geq 5$ years

$28(22.8 \%)$

Notes: aData not available for one patient; bdata expressed as mean \pm standard deviation.

Abbreviation: ICD, International Classification of Diseases.

medical care (87\%); 9.8\% had specialized outpatient palliative care.

The majority of the patients were in constant pain (93.5\%), and 35.8\% had additional breakthrough pain. Most patients (75.6\%) had pain duration of at least 1 year; for $22.8 \%$, more than 5 years were documented. A total of $74 \%$ of the patients suffered from mixed pain, $7.3 \%$ from predominantly nociceptive, and $9.8 \%$ from predominantly neuropathic pain. Main underlying pain etiology was the tumor (50.4\%) and side effects of cancer therapy (16.3\%). A total of $51.2 \%$ of the patients had been hospitalized owing to pain during the 6 months prior to the start of tapentadol PR treatment.

At baseline evaluation, all patients were already under long-term analgesic treatment, mainly consisting of several analgesics: $42.3 \%$ received World Health Organization (WHO) step III opioids, 39\% WHO II opioids, and 78.9\% nonopioids. The use of antidepressants (26.8\%), antiepileptics (18.7\%), muscle relaxants (14.6\%), laxatives $(22.8 \%)$, and antiemetics $(16.3 \%)$, as well as breakthrough pain medication (20.3\%), was also documented. Evaluation of health state by the patients (EQ-5D-3L) showed marked pain-related restrictions in mobility (73.9\% of the patients), self-care $(62.6 \%)$, daily activities $(92.7 \%)$, pain/discomfort $(100 \%)$, and anxiety/depression (87\%), associated with severe impairments in quality of life (75.3\%, QLIP). 


\section{Tapentadol PR treatment}

The most common reasons for switching to tapentadol PR were insufficient analgesic efficacy (85.4\%) and insufficient quality of life $(58.5 \%)$. The majority of the patients $(69.1 \%)$ started treatment with $2 \times 50 \mathrm{mg}$ tapentadol $\mathrm{PR} /$ day; $22.8 \%$ received $2 \times 100 \mathrm{mg}$ /day and $8.1 \%$ at least $2 \times 150 \mathrm{mg} /$ day. The mean daily dosage was $141.8 \pm 75.6 \mathrm{mg}$ at start of treatment, $267.0 \pm 126.6 \mathrm{mg}$ at treatment follow-up, and $286.7 \pm 139.8 \mathrm{mg}$ at the end of the 3-month observation. Median treatment duration was 85 days. Two-thirds of the patients $(69.9 \%)$ continued tapentadol treatment; the main reasons for treatment discontinuation were death because of underlying disease progression (9.8\%), insufficient pain relief $(7.3 \%)$, and side effects $(2.4 \%)$.

At treatment initiation with tapentadol PR, $10.6 \%$ of the patients received strong opioids as additional long-term medication, $7.3 \%$ received weak opioids, and $64.2 \%$ nonopioids. At the end of observation, $13 \%$ were administered additional strong opioids, $2.4 \%$ weak opioids, and $47.2 \%$ nonopioids. Both at the start of treatment and at the end of observation, $29.3 \%$ of the patients reported taking additional analgesic medication as needed.

\section{Pain reduction}

Mean average pain intensity at baseline visit was $6.1 \pm 1.7$ points; $84.6 \%$ of the patients had a pain score $\geq 5$ (NRS-11). The mean individual treatment target was stipulated as an average pain intensity of $2.8 \pm 1.6$. All three mean pain intensities were significantly reduced under tapentadol PR (Figure 1): -2.1 (95\% confidence interval [CI] -2.6 to -1.7 ) for the lowest, $-2.4(95 \% \mathrm{CI}-2.8$ to -2.0$)$ for the average, and $-2.9(95 \% \mathrm{CI}-3.3$ to -2.4$)$ for the highest pain intensity (each $P<0.001)$. Half of the patients (52\%) achieved a pain score $\leq 3$ at the end of observation, $41.5 \%$ achieved their individual treatment target, and $33.3 \%$ achieved $>50 \%$ reduction in pain intensity compared to baseline (the results of the responder analysis are summarized in Table 2). Fewer patients had breakthrough pain $(27.6 \%$ versus $35.8 \%$ at baseline), and the mean number of pain episodes decreased from $22.2 \pm 15.5$ to $10.4 \pm 12.3$ per week.

\section{Anxiety and depression}

Information about the anxiety/depression status of the patients was obtained with two questionnaires: the EQ5D-3L and the HADS. After 3-month tapentadol treatment, the proportion of patients experiencing pain-related anxiety or depression decreased from $87 \%$ at baseline to $62.6 \%$ (EQ-5D-3L). Compared to baseline (31.7\%) only $8.1 \%$ were still extremely anxious or depressed. Analysis of the HADS questionnaire also showed a reduction in anxiety and depression (Table 3). Patients were also asked about suicidal thoughts. Nine of 36 patients $(25 \%)$ who answered this question reported thinking occasionally or frequently about suicide; at the end of observation, the proportion had decreased to $8.3 \%$ (five of $60, \mathrm{LOCF}$ ).

\section{Pain-related impairments in daily life and assessment of quality of life}

At the end of observation, pain-related restrictions in daily activities and in sleep had been markedly reduced (Figure 2). Overall, the mean mPDI sum score improved from 45.6 \pm 14.0

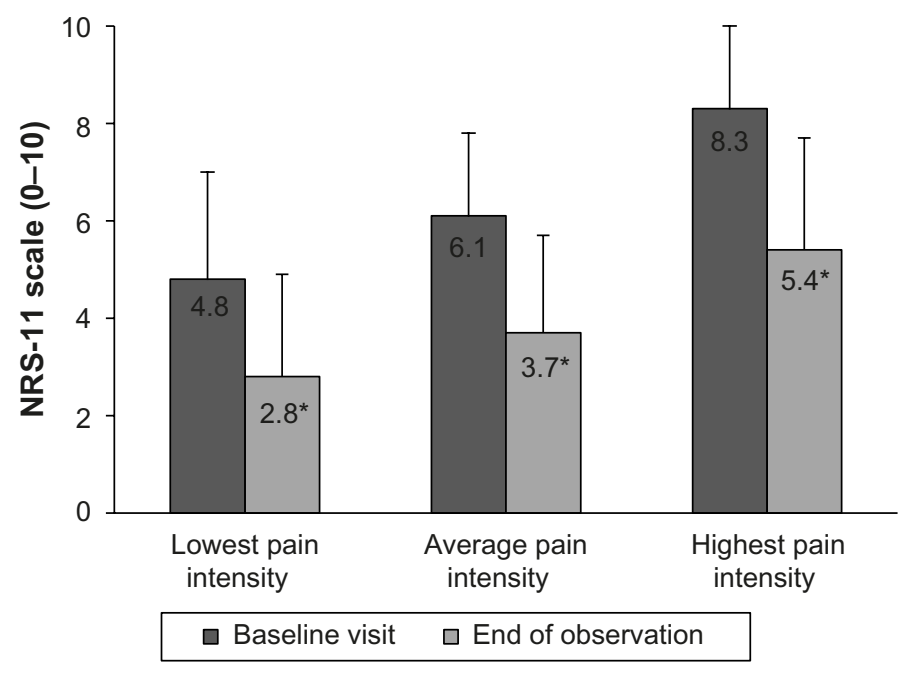

Figure I Reduction of pain intensity under tapentadol prolonged release during the 3-month observation period.

Notes: $* \mathrm{p}<0.001$ (descriptive, paired $t$-test). Data are mean \pm standard deviation. Baseline visit $\mathrm{n}=122$, end of observation $\mathrm{n}=1 \mid \mathrm{I}$ (lowest), $\mathrm{n}=1 \mathrm{I0}$ (average), and $\mathrm{n}=109$ (highest) pain intensity. NRS-II scale: from $0=$ no pain to $10=$ worst imaginable pain.

Abbreviation: NRS-I I, II-point numerical rating scale. 
Table 2 Responder analysis. Patients experiencing treatment success regarding pain reduction (parameter average pain intensity, $n=123$ )

\begin{tabular}{ll}
\hline Response criteria & $\begin{array}{l}\text { Number of } \\
\text { patients (\%) }\end{array}$ \\
\hline Pain-intensity score of $\leq 3$ (NRS-I I) & $64(52)$ \\
Pain-free state (score of 0, NRS-I I) & $3(2.4)$ \\
Attainment of individual treatment target & 5 I (4I.5) \\
Pain reduction of $\geq 2$ points (NRS- I I) compared & $78(63.4)$ \\
to baseline & \\
Pain reduction $>30 \%$ compared to baseline & $72(58.5)$ \\
Pain reduction $>50 \%$ compared to baseline & 4 I (33.3) \\
Pain reduction $>70 \%$ compared to baseline & I5 (I2.2) \\
\hline
\end{tabular}

Abbreviation: NRS-I I, I I-point numerical rating scale.

to $30.4 \pm 20.4$ points, and the proportion of patients severely affected (score $>43$ ) was reduced from $54.5 \%$ to $32.5 \%$. The number of patients without impairments also increased in the dimensions of the EQ-5D-3L: from $26 \%$ to $43.1 \%$ for mobility, from $36.6 \%$ to $50.4 \%$ for self-care, from 0 to $10.6 \%$ for pain/discomfort, and from $7.3 \%$ to $27.6 \%$ for daily activities. The overall rating of the EQ-5D-3L showed a significant improvement of a mean 1.5 points $(95 \% \mathrm{CI}-1.9$ to $-1.1, P<0.001)$ compared to baseline (10.3 \pm 1.8$)$, and the general health state of the patients improved significantly from $63.5 \pm 14.9$ to $45.9 \pm 23.9$ points $(P<0.001)$.

Pain-related impairment of quality of life was also markedly reduced. The mean mPDI-7 (impairment of overall quality of life) improved from 7.0 2 2.3 at baseline to $4.2 \pm 2.9$ (Figure 2). Mean impairment of overall quality of life in particular showed the greatest improvement (representative for all individually determined mean impairments over the course of tapentadol treatment). The proportion of patients with severe impairments (score $>7$ ) decreased from $47.9 \%$ to $12.5 \%$. Furthermore, evaluation with the QLIP inventory showed an improvement in the proportion of patients with abnormal scores (sum score $\leq 20$ ) from $75.3 \%$ to $61 \%$, and both physical and mental component scores of the SF-12 had increased at the end of observation (Table 3). Mean overall habitual well-being (determined with the MFHW) improved from $1.1 \pm 0.8$ to $1.7 \pm 1.4$ points (Table 3 ).

Overall, physicians and patients rated tapentadol treatment as similarly positive: the overall condition of the patients since the start of treatment was considered as "improved" to "very much improved" by both groups (physicians $81.3 \%$, patients $80.7 \%$; Figure 3 ). According to the physicians, in particular effectiveness of treatment (79.8\%), quality of life $(69.7 \%)$, overall treatment success $(56 \%)$, overall tolerability of the treatment (55\%), gastrointestinal tolerability (44\%), and balance between efficacy and tolerability (38.5\%) had improved in comparison to previous analgesic treatment (data available for 109 of the 123 patients). Furthermore, concomitant analgesic medication could be reduced in $38.5 \%$ of the patients.

\section{Safety}

Thirty-two ADRs were noted for 15 of the 123 patients (12.2\%); one ADR (nausea) was rated as serious. There were no further serious ADRs. Four ADRs (abdominal distention, abdominal pain, $\gamma$-glutamyl transferase increased, bladder

Table 3 Changes in quality of life and mental state during the 3-month observation (LOCF data, $\mathrm{n}=123$ )

\begin{tabular}{|c|c|c|}
\hline & Baseline & End of observation \\
\hline \multicolumn{3}{|l|}{ Anxiety and depression (HADS) } \\
\hline Anxiety (sum score) & II.I $\pm 4.8(10.0-12.3)$ & $7.6 \pm 5.0(6.5-8.9)$ \\
\hline Proportion of moderately to severely affected patients & $48.1 \%$ & $31.2 \%$ \\
\hline Depression (sum score) & $13.0 \pm 4.9(|1.9-| 4.2)$ & $8.8 \pm 5.6(7.5-10.1)$ \\
\hline Proportion of moderately to severely affected patients & $66.2 \%$ & $37.7 \%$ \\
\hline Habitual well-being (MFHW) & $1.1 \pm 0.8(0.9-1.3)$ & $1.7 \pm 1.4(1.4-2.0)$ \\
\hline Proportion of patients with a score $<2.5$ & $76.6 \%$ & $63.6 \%$ \\
\hline \multicolumn{3}{|l|}{ Quality of life } \\
\hline QLIP sum score & $11.6 \pm 7.3(9.9-13.3)$ & $15.7 \pm 9.1(13.6-17.8)$ \\
\hline Proportion of severely affected patients & $75.3 \%$ & $61 \%$ \\
\hline mPDI-7 (overall quality of life) & $7.0 \pm 2.3(6.5-7.6)$ & $4.2 \pm 3.4(3.5-5.0)$ \\
\hline \multicolumn{3}{|l|}{ SF-12 } \\
\hline Physical component score & $27.7 \pm 6.2(26.1-29.3)$ & $36.1 \pm 10.9(33.5-38.8$ \\
\hline Mental component score & $33.2 \pm 9.9(30.9-35.4)$ & $40.8 \pm 12.2(38.1-43.6)$ \\
\hline
\end{tabular}

Notes: Data are mean \pm standard deviation ( $95 \%$ confidence interval) or number of patients (\%). SF-12 German population norms are 49.6 for the average physical sum score and 52.3 for the average mental sum score. HADS: sum score $\mid-7=$ normal, $8-10=$ mildly affected, ||$-2 \mid=$ moderately to severely affected. MFHW: $0=$ worst possible state to $5=$ best possible state; $<2.5=$ abnormal. mPDI: from $0=$ no impairment to $10=$ total impairment. QLIP: sum score from $0=$ most affected to $40=$ not affected; $\leq 20=$ severely affected. Abbreviations: LOCF, last observation carried forward; HADS, Hospital Anxiety and Depression Scale; MFHW, Marburger Fragebogen zum habituellen Wohlbefinden [Marburg questionnaire on habitual health findings]; mPDI, modified Pain Disability Index; QLIP, Quality of Life Impairment by Pain inventory; SF, short form. 


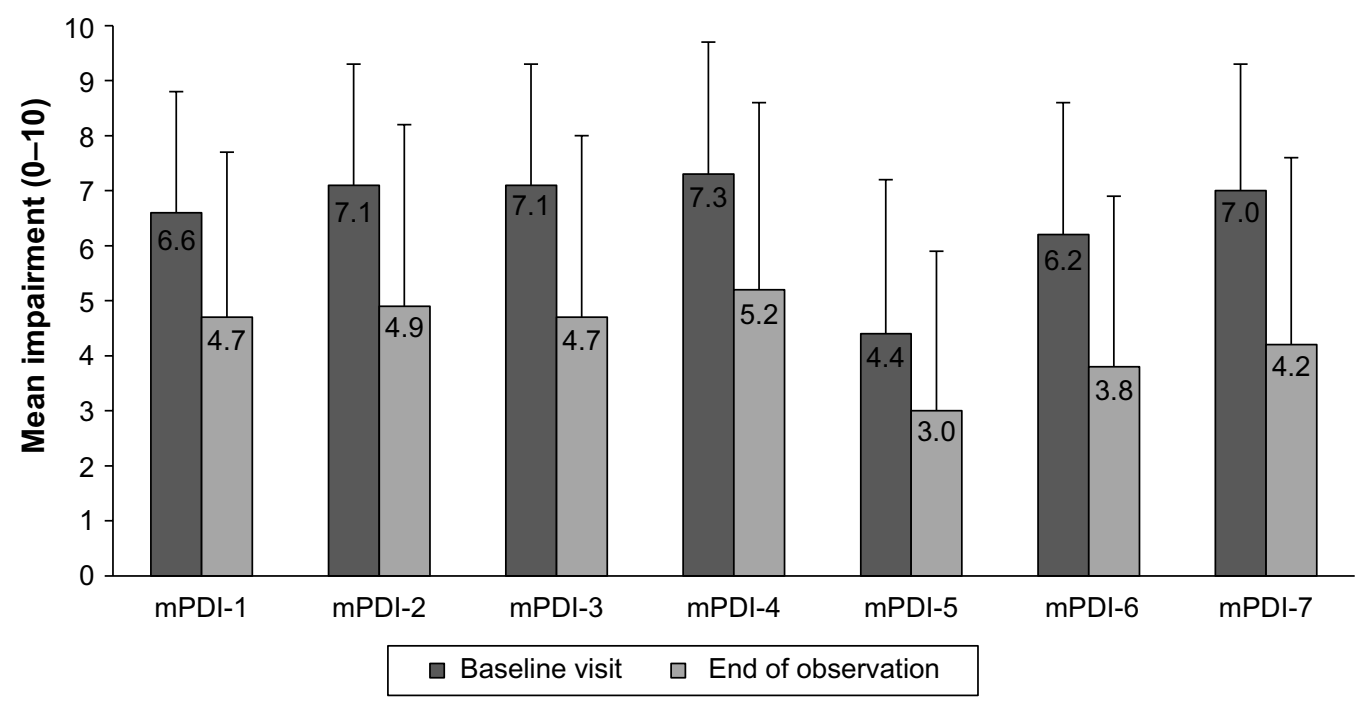

Figure 2 Changes in pain-related impairments in daily life under tapentadol prolonged release (LOCF data, $\mathrm{n}=123$ ).

Notes: Data are mean \pm standard deviation. Rating scale from $0=$ no impairment to $10=$ total impairment. mPDI- I, related to home and family; mPDI-2, recreational activities; mPDI-3, social activities; mPDI-4, occupational activities including housework or volunteering; mPDI-5, self-care; mPDI-6, sleep; mPDI-7, overall quality of life.

Abbreviations: LOCF, last observation carried forward; mPDI, modified Pain Disability Index.

pain) were assessed as unlabeled for tapentadol PR. For these ADRs, alternative explanations (medical history, concomitant medication) were reported. The most frequent ADRs concerned the system-organ classes of gastrointestinal (eleven) and nervous system (nine) disorders. Most frequent were nausea and dizziness (four each) and vomiting and fatigue (two each). Overall, treatment with tapentadol PR was well tolerated.

\section{Discussion}

Half of the cancer patients mainly treated by palliative care specialists were at an advanced metastatic disease stage; a third were either entirely in need of care or - if not bedriddenat least dependent on some nursing or physician assistance. The vast majority had suffered for a long time from severe chronic pain that could not be sufficiently controlled despite long-term treatment with several, often strong analgesics
( $42 \%$ of the patients received WHO III medication). This was accompanied by partly marked impairments in daily life and social interactions, anxiety, depression, and a reduced quality of life.

The 3-month treatment with tapentadol PR resulted in significant pain reductions. A total of $41.5 \%$ of the patients attained their individual treatment target, and more than half $(52 \%)$ achieved a pain score $\leq 3$ at the end of observation. Breakthrough pain was less frequent than at the start of treatment. Pain relief in this patient cohort was not quite as pronounced as in cancer patients treated in general or internal medicine practice ( -2.4 versus -3.8 points).${ }^{17} \mathrm{~A}$ comparison between these two different study populations is however difficult, because there were no data regarding tumor staging and disease progression available for patients treated primarily by general practitioners or specialists for internal

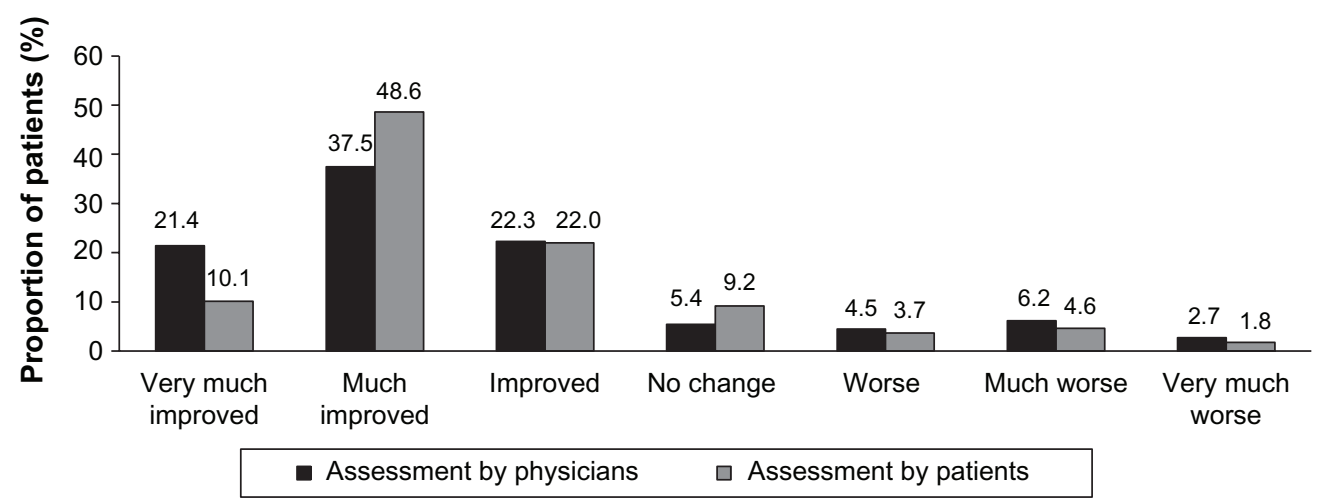

Figure 3 Change in the general condition of the patients since treatment initiation with tapentadol prolonged release. Ratings were available for II 2 patients (physician rating) and for 109 patients (patient rating). 
medicine in the former study. The general condition of its patient population can thus not be evaluated.

Apart from pain control, improvements of pain-related restrictions in daily life and the preservation or restoration of personal independence and self-care are important treatment issues. Uninterrupted sleep, independent personal hygiene, participation in daily events, and social interaction improve the physical and emotional well-being and thus individual quality of life of the patients. In particular for patients with advanced cancer, preservation of quality of life is an important aspect of therapy. Pain-related burden increases with pain intensity ${ }^{3}$ an increase according to grade of chronification was additionally described for nonmalignant pain. ${ }^{28}$

Treatment with tapentadol demonstrated good effectiveness and tolerability over the 3-month observation in this patient population suffering from severe chronic cancerrelated pain; the administration of concomitant analgesic medication decreased overall, cancer pain-related mental and physical burden was markedly reduced, and the general health state of the patients improved significantly despite the underlying illness. Anxiety disorders and depression were reduced, fewer patients thought about suicide, and the proportion of patients with severe impairments of quality of life decreased. The aspect "pain" obviously plays an important role in the overall well-being and quality of life of these patients, and might - if adequately treated - alleviate the suffering owing to the cancer. Adequate pain control accompanied by good tolerability allows the treating physician to focus consultation visits on the treatment of the underlying illness.

The majority of cancer patients suffered from mixed pain $(74 \%)$. In the treatment of pain with a nociceptive and a neuropathic component, targeting both the $\mu$-opioid receptor and the noradrenaline reuptake might be an advantage. In contrast to classical opioids that act only as MOR agonists, tapentadol combines both MOR and NRI mechanisms of action. Tapentadol has been proven effective in nociceptive and neuropathic pain conditions. ${ }^{8}$ In the management of low-back pain, an often mixed-pain syndrome, tapentadol was effective in patients with and without a neuropathic pain component. ${ }^{29}$ The substantial reductions in pain intensity and pain-related impairments shown in the present patient population indicate the suitability of tapentadol for the treatment of mixed cancer pain.

Compared to the observation of cancer patients in general and internal medicine practice, ${ }^{17}$ dosages of tapentadol PR were higher in the present study; however, maximum permitted dosages (500 mg/day) were not used. The daily dose at the end of this 3-month observation was $287 \pm 140 \mathrm{mg}$ compared to $223 \pm 111 \mathrm{mg}$ in the former study. ${ }^{17}$ Daily dosages in clinical studies on cancer pain treatment varied depending on the patient population, with a mean daily $190 \pm 114 \mathrm{mg}$ for opioid-naïve patients, ${ }^{12}$ as well as median $64.5 \mathrm{mg},{ }^{13}$ and median modal dosages of $300 \mathrm{mg}$ for moderate or severe pain. ${ }^{14}$ In the present study, overall fewer patients received weak opioids and nonopioids concomitant to tapentadol at the end of observation; the proportion of patients on strong opioids increased slightly. A possible reason was the relatively low level of experience of the participating physicians at the time of the switch to tapentadol in this study, since tapentadol had only received market authorization in 2010.

Physicians noted insufficient analgesia with previous opioids as the main reason for switching to tapentadol for the majority of the patients. They prescribed other strong or weak opioids or nonopioids as additional long-term analgesics to tapentadol therapy, if needed. The administration of concomitant analgesics had decreased overall at the end of observation, whereas pain severity and pain-related impairments had improved. This indicates the effectiveness of tapentadol as the main analgesic medication.

When evaluating these data, it should be taken into account that the study design was a noninterventional observation without a control group. The return rate of the pain questionnaire/pain diary was low; it should however be noted that we were able to document comprehensively the mental and physical situation of the patients because we used a variety of standardized instruments. Furthermore, our study design complements randomized clinical studies (with a predefined protocol and strict inclusion and exclusion criteria) and reflects routine clinical treatment practice for a broad spectrum of patients suffering from cancer-related pain.

\section{Conclusion}

Tapentadol PR is an effective and well-tolerated treatment option for severe chronic cancer-related pain. In addition to good pain reduction and good tolerability of the medication, pain-related mental and physical burden was reduced and quality of life improved. The overall health state of the cancer pain patients significantly improved despite the underlying illness; this might alleviate the suffering caused by the disease.

\section{Acknowledgments}

The authors thank all participating physicians; and Elke Grosselindemann and Birgit Brett for writing and editorial assistance. Grünenthal $\mathrm{GmbH}$, Aachen, Germany supported 
the study and met all costs in connection with the preparation of the manuscript.

\section{Author contributions}

DK and KS were involved in study design and data analysis; AS was involved in data acquisition. All authors contributed toward data interpretation, critically revised the manuscript, and agree to be accountable for all aspects of the work. All approved the final manuscript version.

\section{Disclosure}

AS is a consultant for Grünenthal $\mathrm{GmbH}$. He participated in the study and received financial recompense for complete documentation of the patients. DK and KS are employees of Grünenthal GmbH. The authors report no other conflicts of interest in this work.

\section{References}

1. van den Beuken-van Everdingen MH, de Rijke JM, Kessels AG, Schouten $\mathrm{HC}$, van Kleef M, Patijn J. Prevalence of pain in patients with cancer: a systematic review of the past 40 years. Ann Oncol. 2007;18:1437-1449.

2. Portenoy RK. Treatment of cancer pain. Lancet. 2011;377: 2236-2247.

3. te Boveldt N, Vernooij-Dassen M, Burger N, Ijsseldijk M, Vissers K, Engels Y. Pain and its interference with daily activities in medical oncology outpatients. Pain Physician. 2013;16:379-389.

4. Caraceni A, Hanks G, Kaasa S, et al. Use of opioid analgesics in the treatment of cancer pain: evidence-based recommendations from the EAPC. Lancet Oncol. 2012;13:e58-e68.

5. Bennett MI, Graham J, Schmidt-Hansen M, Prettyjohns M, Arnold S. Prescribing strong opioids for pain in adult palliative care: summary of NICE guidance. BMJ. 2012;344:e2806.

6. Benyamin R, Trescot AM, Datta S, et al. Opioid complications and side effects. Pain Physician. 2008;11:S105-S120.

7. Tzschentke TM, Christoph T, Kögel BY. The mu-opioid receptor agonist/noradrenaline reuptake inhibition (MOR-NRI) concept in analgesia: the case of tapentadol. CNS Drugs. 2014;28:319-329.

8. Taylor R, Pergolizzi JV, Raffa RB. Tapentadol extended release for chronic pain patients. Adv Ther. 2013;30:14-27.

9. Merker M, Dinges G, Koch T, Kranke P, Morin AM. [Undesired side effects of tapentadol in comparison to oxycodone. A meta-analysis of randomized controlled comparative studies]. Schmerz. 2012;26:16-26. German.

10. Schwittay A, Schumann C, Litzenburger B, Schwenke K. Tapentadol prolonged release for severe chronic pain: results of a noninterventional study involving general practitioners and internists. J Pain Palliat Care Pharmacother. 2013;27:225-234.

11. Strohmeier M, Waldmann-Rex S, Schwenke K. [Influence of tapentadol retard on functional parameters in patients with severe chronic pain -is there a benefit in daily life?] MMW Fortschr Med. 2013;155: 63-71. German.

Journal of Pain Research

\section{Publish your work in this journal}

The Journal of Pain Research is an international, peer-reviewed, open access, online journal that welcomes laboratory and clinical findings in the fields of pain research and the prevention and management of pain. Original research, reviews, symposium reports, hypothesis formation and commentaries are all considered for publication.

Submit your manuscript here: http://www.dovepress.com/journal-of-pain-research-journal
12. Mercadante S, Porzio G, Ferrera P, et al. Tapentadol in cancer pain management: a prospective open-label study. Curr Med Res Opin. 2012;28:1775-1779.

13. Imanaka K, Tominaga Y, Etropolski M, et al. Efficacy and safety of oral tapentadol extended release in Japanese and Korean patients with moderate to severe, chronic malignant tumor-related pain. Curr Med Res Opin. 2013;29:1399-1409.

14. Kress HG, Koch ED, Kosturski H, et al. Tapentadol prolonged release for managing moderate to severe, chronic malignant tumor-related pain. Pain Physician. 2014;17:329-343.

15. Mercadante S, Porzio G, Adile C, et al. Tapentadol at medium to high doses in patients previously receiving strong opioids for the management of cancer pain. Curr Med Res Opin. 2014;30:2063-2068.

16. Imanaka K, Tominaga Y, Etropolski M, Ohashi H, Hirose K, Matsumura T. Ready conversion of patients with well-controlled, moderate to severe, chronic malignant tumor-related pain on other opioids to tapentadol extended release. Clin Drug Investig. 2014;34: 501-511.

17. Agbalaka A, Schwenke K, Litzenburger L. [Tapentadol prolonged release for the treatment of severe chronic tumor pain in routine clinical practice]. MMW Fortschr Med. 2012;154:123-130. German.

18. Grünenthal GmbH. Fachinformation Palexia ${ }^{\circledR}$ retard [German summary of product characteristics]. Aachen, Germany: Grünenthal; 2010.

19. Dworkin RH, Turk DC, Farrar JT, et al. Core outcome measures for chronic pain clinical trials: IMMPACT recommendations. Pain. 2005;113:9-19.

20. Karnovsky DA, Burchenal JH. The clinical evaluation of chemotherapeutic agents in cancer. In: MacLeod CM, editor. Evaluation of Chemotherapeutic Agents. New York: Columbia University Press; 1949:196.

21. Oken MM, Creech RH, Tormey DC, et al. Toxicity and response criteria of the Eastern Cooperative Oncology Group. Am J Clin Oncol. 1982;5:649-655.

22. EuroQoL. How to use EQ-5D. Available from: http://www.euroqol. org/about-eq-5d/how-to-use-eq-5d.html. Accessed May 15, 2014.

23. Deutsche Schmerzgesellschaft. Deutscher Schmerzfragebogen [German pain questionnaire]. Available from: http://www.dgss.org/deutscherschmerzfragebogen. Accessed May 15, 2014.

24. Tait RC, Chibnall JT, Krause S. The pain disability index: psychometric properties. Pain. 1990;40:171-182.

25. Basler HD. [The Marburg questionnaire on habitual health findings a study on patients with chronic pain]. Schmerz. 1999;13:385-391. German.

26. Zigmond AS, Snaith RP. The hospital anxiety and depression scale. Acta Psychiatr Scand. 1983;67:361-370.

27. Ware J Jr, Kosinski M, Keller SD. A 12-item short-form health survey: construction of scales and preliminary tests of reliability and validity. Med Care. 1996;34:220-233.

28. Frettlöh J, Maier C, Gockel H, Hüppe M. [Validation of the German Mainz Pain Staging System in different pain syndromes]. Schmerz. 2003;17:240-251. German.

29. Steigerwald I, Müller M, Davies A, et al. Effectiveness and safety of tapentadol prolonged release for severe, chronic low back pain with or without a neuropathic pain component: results of an open-label, phase $3 \mathrm{~b}$ study. Curr Med Res Opin. 2012;28:911-936.

\section{Dovepress}

The manuscript management system is completely online and includes a very quick and fair peer-review system, which is all easy to use. Visit http://www.dovepress.com/testimonials.php to read real quotes from published authors. 